\title{
An Integro-Differential Model for Non-Fickian tracer transport in porous media: validation and numerical simulation
}

\author{
J. A. Ferreira and L. Pinto \\ CMUC, Department of Mathematics, University of Coimbra, \\ 3001-454 Coimbra, Portugal.
}

\begin{abstract}
Diffusion processes have traditionally been modeled using the classical parabolic advection-diffusion equation (ADE). However, as in the case of tracer transport in porous media, significant discrepancies between experimental results and numerical simulations have been reported in the literature. Therefore, in order to describe such anomalous behavior, known as non-Fickian diffusion, some authors have replaced the parabolic model with the continuous random walk model (CTRW), which has been very effective. Integro-differential models (IDMs) have been also proposed to describe non-Fickian diffusion in porous media. In this paper, we introduce and test a particular type of IDM by fitting breakthrough curves (BTCs) resulting from laboratory tracer transport. Comparisons with the traditional ADE and the CTRW are also presented. Moreover, we propose and numerically analyze a stable and accurate numerical procedure for the two-dimensional IDM composed by a integro-differential equation for the concentration and Darcy's law for flow. In space, it is based on the combination of mixed finite element (MFE) and finite volume methods over an unstructured triangular mesh.
\end{abstract}

Keywords: Tracer transport; Porous media; Non-Fickian; Integrodifferential; CTRW; Validation; Numerical simulation.

\section{Introduction}

A wide range of physical, chemical, and biological phenomena in porous media are traditionally modeled by advection-diffusion equations. For instance, the important problem of non-reactive tracer transport in porous media, can be described by the following classical ADE

$$
\phi \frac{\partial u}{\partial t}+\nabla \cdot(u v)=\nabla \cdot(D(v) \nabla u)+q u^{*}
$$


where $\phi$ denotes the porosity of the medium, $u$ the concentration of the tracer, $v$ the fluid velocity, $q$ source and sinks terms, and $u^{*}$ a prescribed concentration at sources or $u$ at sinks. The dispersion tensor $D(v)$ is given by

$$
D(v)=d_{m} \phi I+\alpha_{t}\|v\| I+\left(\alpha_{\ell}-\alpha_{t}\right) \frac{1}{\|v\|} v v^{T},
$$

where $I$ is the identity tensor, $d_{m}$ the molecular diffusion coefficient, and $\alpha_{\ell}$ and $\alpha_{t}$ the longitudinal and transverse dispersivities, respectively. When the velocity field $v$ is unknown, the equation (1) is closed by taking into account Darcy's law, which establishes a relation between the pressure gradient and velocity $[1,2,3,4,5]$. The $\mathrm{ADE}(1)$ can be derived from the mass conservation equation

$$
\phi \frac{\partial u}{\partial t}+\nabla \cdot J=q u^{*},
$$

where the total mass flux $J$ is given by

$$
J=J_{a d v}+J_{d i s},
$$

where $J_{a d v}$ is the flux due to convective transport

$$
J_{a d v}=v u,
$$

and, by assuming that the diffusion-dispersion mass flux $J_{d i s}$ satisfies the socalled Fick's law,

$$
J_{d i s}=-D(v) \nabla u .
$$

The equation (1), being of the parabolic type, induces a pathologic behavior in the concentration $u$; namely, it presents infinite speed of propagation which has no physical meaning. Other limitations associated with the definition of $D(v)$ have also been reported $[6,7,8]$. Regarding the use of such an equation to simulate tracer transport, several gaps have been observed when simulation results were compared with laboratory experiments. These observations have been extensively reviewed $[9,10,11,12,13,14,15,16,17,18]$.

Several approaches have been developed in order to overcome the limitations associated with (1); we refer, e.g., to [7] and [8]. A common approach consists of the introduction of a hyperbolic or non-Fickian correction term. A particular model of this type can be obtained by assuming that the diffusion-dispersion mass flux $J_{d i s}$ satisfies the following differential equation

$$
\tau \frac{\partial J_{d i s}}{\partial t}(x, t)+J_{d i s}(x, t)=-D(v) \nabla u(x, t),
$$

where $\tau$ is a delay parameter [19]. Note that the left hand side of (5) is a first order approximation of the left hand side of $J_{\text {dis }}(x, t+\tau)=-D(v) \nabla u(x, t)$, which means that the dispersion mass flux at the point $x$ and time $t+\tau$ depends on the gradient of the concentration at the same point but at a delayed time. Equation (5) leads to

$$
J_{d i s}(t)=-\int_{0}^{t} K_{e r}(t-s) D(v) \nabla u(s) d s,
$$


with the kernel term $K_{e r}(t)=\frac{1}{\tau} e^{-\frac{t}{\tau}}$, provided that $J_{d i s}(0)=0$. Combining the partition (4) with (3), where $J_{d i s}$ is given by (6), we obtain the integrodifferential equation

$$
\phi \frac{\partial u}{\partial t}+\nabla \cdot(u v)=\int_{0}^{t} K_{e r}(t-s) \nabla \cdot(D(v) \nabla u)(s) d s .
$$

Moreover, if we assume that the diffusion-dispersion mass flux has both a Fickian component $J_{F}$, and a non-Fickian component $J_{N F}$, defined by

$$
J_{F}(x, t)=-D_{F} \nabla u(x, t),
$$

and

$$
J_{N F}(x, t)=-\int_{0}^{t} K_{e r}(t-s) D_{N F} \nabla u(x, s) d s,
$$

then the ADE (1) is replaced by

$$
\phi \frac{\partial u}{\partial t}+\nabla \cdot(u v)-\nabla \cdot\left(D_{F} \nabla u\right)=\int_{0}^{t} K_{e r}(t-s) \nabla \cdot\left(D_{N F} \nabla u\right)(s) d s+q u^{*} .
$$

The mathematical and numerical analysis of initial boundary value problems (IBVPs) based on integro-differential equations of this type were studied, e.g., in [20], [21], [22], [23], [24], [25] and [26].

The ability of the IDM (9) to capture the dynamics of tracer transport has already been tested by the authors in [27] by fitting the model to experimental BTCs. There a comparison with the well-known CTRW [9, 10, 11, 28, 29, 13, 14] is included. In this paper, we test the models against more experimental data, and the discussion of the results is presented in Section 2. Based on the results of the previous and present studies, we conclude that (9) can be used to accurately describe tracer transport in porous media. In Section 3, with the objective to simulate real-world problems, we coupled (9) with Darcy's equation, and developed an efficient discretization scheme in two dimensions for the resulting system. The numerical validation of the scheme is then performed by providing numerical convergence results for three problems with known theoretical solutions. Numerical simulations showing the qualitative behavior of the IDM in more realistic conditions are also presented in Section 3. Finally, in Section 4, we summarize our conclusions.

\section{Model Validation and Comparison}

\subsection{CTRW}

For completeness, we present, in this section, a brief description of the CTRW. In the CTRW perspective, tracer transport can be seen as a series of particle jumps or transitions characterized by waiting times between jumps. The jumps and the waiting times are coupled by a joint probability density function (pdf) 
$\psi$ that describes, at $(x, t)$, the jump at position $x$ and time $t$. When the jumps occur in $\mathbb{R}$, the pdf $\psi$ and $u$ are linked by the following equation

$$
s \phi\left(\tilde{u}(x, s)-u_{0}(x)\right)=-\tilde{M}(s)(\nabla \cdot(\tilde{u}(x, s) v)-\nabla \cdot(D(v) \nabla \tilde{u})(x, s)),
$$

where $\tilde{u}$ denotes the Laplace transform of $u, v=\frac{1}{t_{1}} \int_{\mathbb{R}} p(z) z d z, D=\frac{1}{2 t_{1}} \int_{\mathbb{R}} p(z) z^{2} d z$, and

$$
\tilde{M}(s)=\frac{s t_{1} \tilde{\varphi}(s)}{1-\tilde{\varphi}(s)},
$$

representing $t_{1}$ a median transition time. Equation (10) is obtained by assuming that the Laplace transform $\tilde{\psi}$ of $\psi$ admits a factorization $\tilde{\psi}(x, s)=p(x) \tilde{\varphi}(s)$, where $p$ denotes the transition length pdf and $\varphi$ denotes the marginal density of $\psi$. Notice that (10) also holds if the pdf $\varphi$ has a compact support in $\mathbb{R}$.

A very common CRTW, and the one that we focus on in this paper, is obtained from (10) when $p$ is a Gaussian pdf and $\varphi$ is the truncated power law pdf

$$
\varphi(t)=\frac{\left(1+t / t_{1}\right)^{-1-\beta}}{t_{1} r^{\beta} \Gamma(-\beta, r)} e^{\left(-\frac{t_{1}+t}{t_{2}}\right)}, \quad r=\frac{t_{1}}{t_{2}}, t_{1}<t_{2}, 0 \leq \beta \leq 2,
$$

where $\Gamma$ is the incomplete Gamma function. This particular model has been used widely to simulate tracer transport in porous media [10, 11, 28, 29].

\section{$2.2 \quad$ BTC analysis}

The objective of this section is to test the IDM against real data and to compare its effectiveness with the ADE and CTRW. The analysis was made by comparing BTCs measured in a laboratory environment with those predicted by the three classes of differential equations. This study complements the results presented in [27].

A usual approach in the validation of mathematical models for tracer transport is to consider that the experiments can be described by one-dimension models. Therefore, equations (1), (9), and (10) are defined in $(0, L) \times(0, T]$ and are completed with the initial condition $u(x, t)=u_{0}(x), x \in(0, L)$. As the concentration of the injected tracer is known at the boundary $x=0$, and at $x=L$ the fluid is removed, we assume the following boundary conditions

$$
u(0, t)=u_{i}(t), u(L, t)=0, \quad t \in(0, T]
$$

To compute the numerical solution of the resulting IBVPs, we introduce the grid $\left\{\left(x_{i}, t_{n}\right), i=0, \ldots, N_{x}, n=0, \ldots, N_{t}\right\}$, where $x_{0}=0, x_{N_{x}}=L, x_{i}-x_{i-1}=h$, $t_{0}=0, t_{N_{t}}=T$ and $t_{n}-t_{n-1}=\Delta t$.

We now follow the approach taken in [27]. The numerical BTCs are computed by minimizing the root mean square error (RMSE), which is defined by

$$
R M S E=\left(\frac{1}{M} \sum_{m=1}^{M}\left(u^{m}-u_{h}^{m}\right)^{2}\right)^{1 / 2},
$$


where $M$ is the number of observations, $u^{m}$ the measured concentration at time $t_{m}$ and $u_{h}^{m}$ the estimated concentration at time $t_{m}$. The minimization of the RMSE for (1) and (10) was obtained using the freely available CTRW toolbox [30], while for (9) we used the built-in routines of Matlab (version 7.9.0 (R2009b)), where the approximation $u_{h}^{m}$ is given by the one-dimensional version of the numerical method studied in [20], [24], and [25]. It should be stressed that the outlet boundary condition is imposed far enough from the grid point where the BTC is evaluated.

These three models have already been compared in [27] using data from two laboratory experiments $[9,31]$. The numerical simulations have shown that the classical ADE does not capture the long tails of the BTCs at later times, while the CTWR and the IDM are able to reproduce this non-Fickian behavior, leading the last model to a greater reduction of RMSE. Here, we confront the models with tracer data from a laboratory experiment described in [13]. This was a large-scale experiment, using soil columns with a length of $1250 \mathrm{~cm}$ and a cross section of $10 \times 10 \mathrm{~cm}^{2}$. The tracer tests were conducted under homogeneous and heterogeneous conditions. We only use the data obtained in the heterogeneous column. This column was randomly packed with various soil materials with different shapes and sizes. The study was conducted under saturated

Table 1: Fitting parameters for the models plotted in Figures 1 (a) and (b) and the corresponding RMSE values $(400 \mathrm{~cm})$.

\begin{tabular}{llll}
\hline Parameters & ADE & CTRW & IDM \\
\hline$v(\mathrm{~m} / \mathrm{min})$ & $9.39 \times 10^{-1}$ & 26.61 & $9.30 \times 10^{-1}$ \\
$D\left(\mathrm{~m}^{2} / \mathrm{min}\right)$ & 19.82 & 40.34 & \\
$D_{F}\left(\mathrm{~m}^{2} / \mathrm{min}\right)$ & & & 1.15 \\
$D_{N F}\left(\mathrm{~m}^{2} / \mathrm{min}\right)$ & & & 31.43 \\
$\tau(\min )$ & & 0.95 & 67.71 \\
$\beta$ & & \\
RMSE & $5.63 \times 10^{-2}$ & $3.96 \times 10^{-2}$ & $2.97 \times 10^{-2}$ \\
\hline
\end{tabular}

conditions and at the constant flow rate of $2.39 \times 10^{-1} \mathrm{~cm} / \mathrm{min}$. The estimated porosity was 0.37 , leading to an average velocity of $6.45 \times 10^{-1} \mathrm{~cm} / \mathrm{min}$. The tracer was injected as a step input and the concentration along the column was measured by installing 12 electrical conductivity probes that were $100 \mathrm{~cm}$ apart. The collected data and a detailed analysis of the experiment are given in [13]. Note that because of the difference between the length and diameter of the column, the use of one-dimensional models is justified.

For our study, we consider the BTCs measured at the distances of $400 \mathrm{~cm}$ and $600 \mathrm{~cm}$. Because of the normalization used in [13] to report the results, we set $u_{i}=1$ at the inlet boundary. The best-fit BTCs by the models and the experimental observations are given in Figure 1. We observe that the measured BTCs are highly asymmetric with long tails, and it is clear that these nonFickian features are better captured by the IDM than the ADE. The modeling 


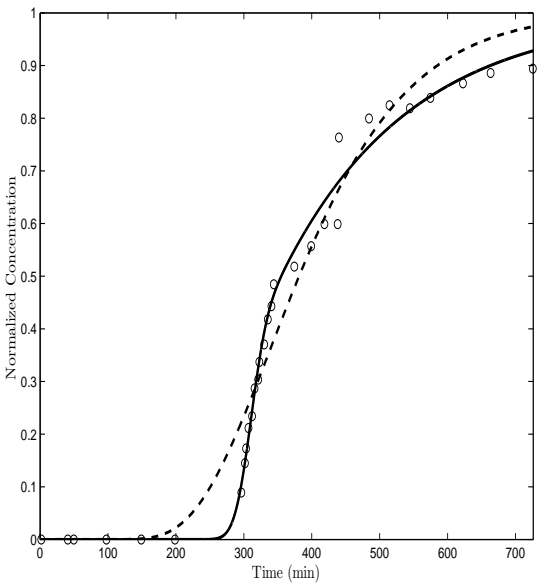

(a) IDM (solid line) and ADE (dash line).

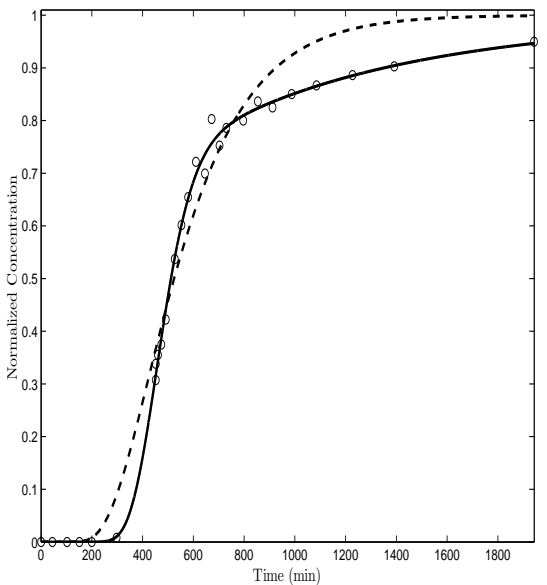

(c) IDM (solid line) and ADE (dash line).

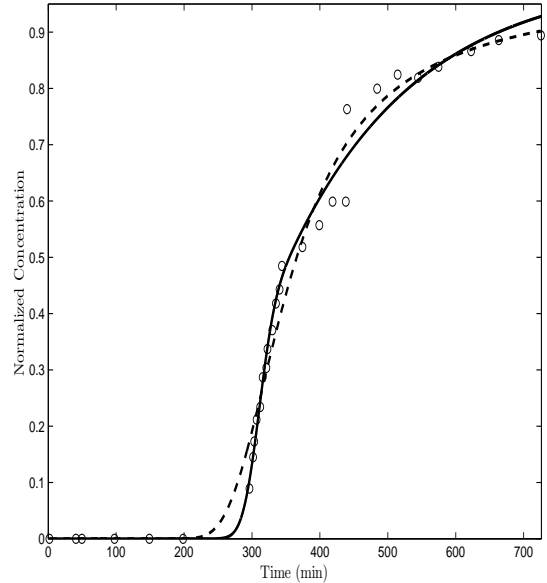

(b) IDM (solid line) and TPL (dash line).

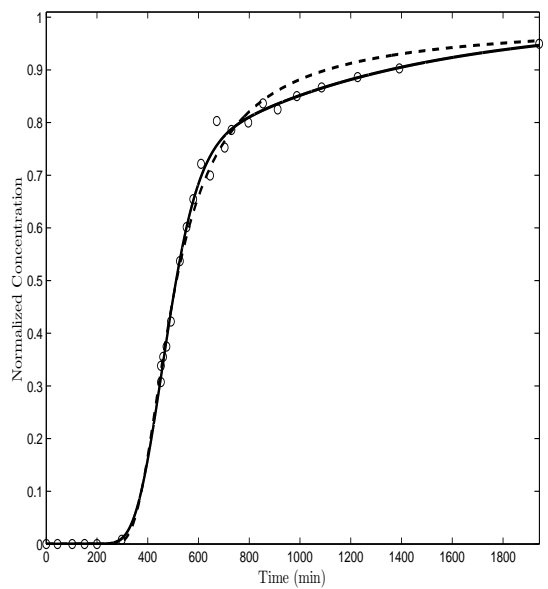

(d) IDM (solid line) and TPL (dash line).

Figure 1: Experimental data and best-fit BTCs at the distances of $400 \mathrm{~cm}$ and $600 \mathrm{~cm}$ away from the inlet in (a) and (b) and in (c) and (d), respectively.

parameters and the RMSEs are shown in Tables 1 and 2. The proposed IDM provided a reduction in the RMSE of $43 \%$ at distance $400 \mathrm{~cm}$ and of $71 \%$ at distance $600 \mathrm{~cm}$. In Figures 1 (b) and (d) we compare again the IDM with the CTRW. As observed in [27], they have a similar performance, but the IDM appears to have smaller RMSE values. This is corroborated by other results not shown here. The same data was used in [29] and [32] to test various transport models including the CTRW.

To conclude this section, we emphasize that, once again, the numerical simulations revealed the superiority of the IDM and CTRW over conventional ADE, 
Table 2: Fitting parameters for the models plotted in Figures 1 (c) and (d) and the corresponding RMSE values $(600 \mathrm{~cm})$.

\begin{tabular}{llll}
\hline Parameters & ADE & CTRW & IDM \\
\hline$v(\mathrm{~m} / \mathrm{min})$ & $9.43 \times 10^{-1}$ & 29.27 & $8.25 \times 10^{-1}$ \\
$D\left(\mathrm{~m}^{2} / \mathrm{min}\right)$ & 50.32 & 70.08 & \\
$D_{F}\left(\mathrm{~m}^{2} / \mathrm{min}\right)$ & & & 20.67 \\
$D_{N F}\left(\mathrm{~m}^{2} / \mathrm{min}\right)$ & & & 187.65 \\
$\tau(\min )$ & & 0.93 & 383.09 \\
$\beta$ & & \\
RMSE & $5.23 \times 10^{-2}$ & $2.30 \times 10^{-2}$ & $1.51 \times 10^{-2}$ \\
\hline
\end{tabular}

leading the IDM to a greater reduction of RMSE.

\subsection{Scale-dependent prediction}

An important aspect that we have not yet discussed is the prediction capacity of the IDM. Our results show that the average velocity is always in good agreement with experiments, suggesting that the mean velocity of the tracer can be correctly predicted. Unfortunately, the other parameters of the model seem to be scale-dependent. For instance, for the data of the experiment reported in [9], and designated Data set 2 in [27] (Section 3.2), the values of $D_{F}$ and $D_{N F}$ clearly increase with distance. A possible solution to mitigate this problem is to insert scale-dependent coefficients in the IDM, similar to those proposed for the $\operatorname{ADE}[33,34,35]$.

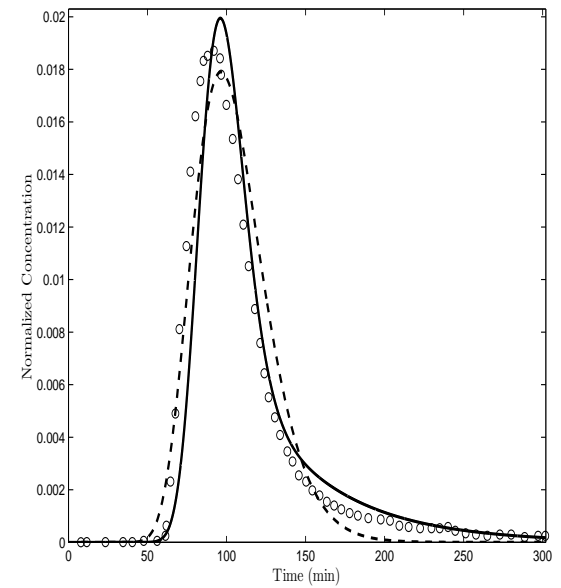

(a) IDM (solid line) and ADE (dash line).

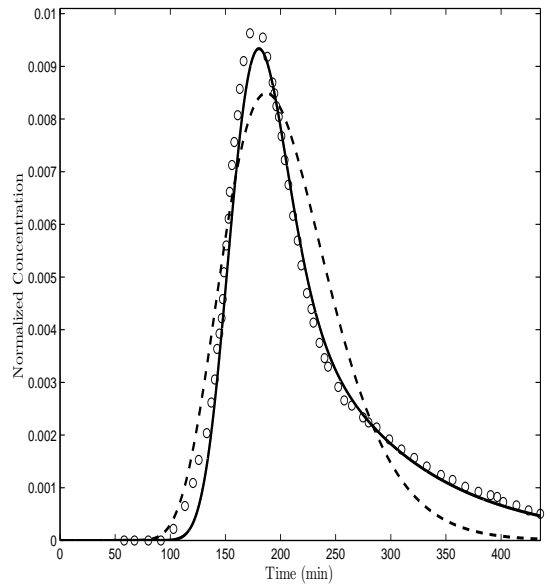

(b) IDM (solid line) and ADE (dash line).

Figure 2: Experimental data and the best-fit predicted BTCs for Column 3 in (a) and for Column 2 in (b). 
For instance, we note that the values of $D, D_{F}$ and $D_{N F}$ in the ADE and IDM seem to vary linearly with the distance $L$, while the value of $\tau$ seem to vary linearly with time $T$. Using the data displayed in Tables 2 and 3 of [27] , we establish the power-law approximations

$$
\begin{aligned}
D_{F} & =2.14 \times 10^{-3} L^{1.08}, & D_{N F} & =9.11 \times 10^{-3} L^{1.04}, \\
\tau & =1.72 \times 10^{-1} T^{0.90}, & D & =2.06 \times 10^{-3} L^{1.28} .
\end{aligned}
$$

From these relations we attempted to predict the BTC for Column 2 and also for another column with $20 \mathrm{~cm}$ length designated Column 3. As it is shown in Figure 2, a good agrement was obtained for the IDM.

\section{Numerical Experiments in Two Dimensions}

\subsection{Governing equations}

The intent of this section is to illustrate the applicability and computational feasibility of the proposed IDM to simulate two-dimensional transport problems. In particular, we are interested in transport problems where the injected tracer and the resident fluid are fully miscible and flow together as a single-phase fluid.

Let $\Omega$ be a bounded domain of $\mathbb{R}^{2}$ representing the porous medium with boundary $\partial \Omega=\partial \Omega_{D} \cup \partial \Omega_{N}$. In $\partial \Omega_{D}$ a Dirichlet boundary condition is prescribed while in $\partial \Omega_{N}$ a mass flux condition is imposed. Assume that $\partial \Omega_{D}=$ $\partial \Omega_{D, i} \cup \partial \Omega_{D, e}$, where $\partial \Omega_{D, i}$ is the inflow boundary and $\partial \Omega_{D, e}$ is the outflow boundary, assume also that no flux occurs through $\partial \Omega_{N}$. In this scenario, the evolution of tracer concentration $u$ will be described by (9), with the initial condition

$$
u(x, 0)=u_{0}(x), \quad x \in \Omega,
$$

and the boundary conditions

$$
\begin{aligned}
u=u_{D} & \text { on } \partial \Omega_{D} \times(0, T], \\
J \cdot \eta=0 & \text { on } \partial \Omega_{N} \times(0, T],
\end{aligned}
$$

where $J$ is the mass flux, as defined in (4), (7), and (8), and $\eta$ is the unit outward vector to $\partial \Omega_{N}$. As the velocity $v$ is unknown, an additional equation is needed. We assume that $v$ satisfies the incompressibility condition

$$
\nabla \cdot v=q \quad \text { in } \Omega,
$$

and obeys Darcy's law

$$
v=-\frac{K}{\mu(u)} \nabla p \quad \text { in } \Omega .
$$

Here $K$ is the permeability tensor and $p$ and $\mu(u)$ are the pressure and viscosity of the fluid mixture, respectively. Equations (14) and (15) are complemented 
by the boundary conditions

$$
\begin{aligned}
p & =p_{D} & & \text { on } \partial \Omega_{D}, \\
v \cdot \eta & =0 & & \text { on } \partial \Omega_{N} .
\end{aligned}
$$

For simplicity, we take in what follows $\mu(u)=1$, i.e., we are assuming that the tracer concentration has no effect on the velocity field. In this way, the coupled problem is reduced to the solution of the IBVP (9), (11)-(13), and the stationary boundary value problem (14)-(17). We consider also that $K, D_{F}$ and $D_{N F}$ are positive defined tensors.

\subsection{A mixed finite element formulation}

The numerical strategy adopted to discretize the uncoupled problem pays special attention to the flow problem (14)-(17). In fact, equation (9) is usually advection dominated and therefore an accurate approximation of $v$ is required. Due to the presence of the tensor $K$ in (15) this can be a challenging task, especially when the medium is highly heterogeneous. We address this issue by using MFEs, a method that has proved very effective for this kind of problems [36, 37, 38, 39]. In particular, we use the lowest-order Raviart-Thomas $\left(R T_{0}\right)$ elements $[40,1,41]$.

Consider the Sobolev space

$$
H(\operatorname{div}, \Omega)=\left\{w \in\left(L^{2}(\Omega)\right)^{2}: \nabla \cdot w \in L^{2}(\Omega)\right\}
$$

and the subspace

$$
V=\left\{w \in H(\operatorname{div}, \Omega): w \cdot \eta=0 \text { on } \partial \Omega_{N}\right\} .
$$

The mixed formulation of the problem (14)-(17) is defined as follows: find $p \in$ $L^{2}(\Omega)$ and $v \in V$ such that

$$
\left(\nabla \cdot v, w_{1}\right)=\left(q, w_{1}\right), \quad \forall w_{1} \in L^{2}(\Omega)
$$

and

$$
\left(K^{-1} v, w_{2}\right)-\left(p, \nabla \cdot w_{2}\right)=-\left(p_{D}, w_{2} \cdot \eta\right)_{\partial \Omega_{D}}, \quad \forall w_{2} \in V,
$$

where $(\cdot, \cdot)_{\partial \Omega_{D}}$ denotes the usual inner product in $L^{2}\left(\partial \Omega_{D}\right)$ and $(\cdot, \cdot)$ denotes the $L^{2}(\Omega)$ or $\left(L^{2}(\Omega)\right)^{2}$ inner product, as appropriate.

The spatial discretization of the IBVP (9), (11)-(13) is also based on a MFE formulation. It reads: find $u(t) \in L^{2}(\Omega)$ and $z(t), \tilde{z}(t) \in V$ such that

$$
\begin{aligned}
& \left(\phi \frac{\partial u}{\partial t}(t), w_{3}\right)+\left(\nabla \cdot(v u(t)), w_{3}\right)+\left(\nabla \cdot z(t), w_{3}\right) \\
& \quad+\int_{0}^{t} K_{e r}(t-s)\left(\nabla \cdot \tilde{z}(s), w_{3}\right) d s=\left(q u^{*}, w_{3}\right),
\end{aligned}
$$

for all $w_{3} \in L^{2}(\Omega)$, and

$$
\begin{aligned}
\left(D_{F}^{-1} z(t), w_{4}\right)-\left(u(t), \nabla \cdot w_{4}\right)=-\left(u_{D}, w_{4} \cdot \eta\right)_{\partial \Omega_{D}}, & \forall w_{4} \in V, \\
\left(D_{N F}^{-1} \tilde{z}(t), w_{5}\right)-\left(u(t), \nabla \cdot w_{5}\right)=-\left(u_{D}, w_{5} \cdot \eta\right)_{\partial \Omega_{D}}, & \forall w_{5} \in V,
\end{aligned}
$$


with

$$
u(0)=u_{0}, \quad \text { in } L^{2}(\Omega)
$$

\subsection{The numerical scheme}

Let $\mathcal{T}_{h}=\left\{T_{i}: i=1, \ldots, N_{T}\right\}$ be an admissible triangulation of the domain $\Omega$ and $\mathcal{E}_{h}=\left\{E_{i}: E_{i} \in \Omega \cup \partial \Omega, i=1, \ldots, M_{E}\right\}$, the set of edges associated with the triangulation. We introduce the elements $R T_{0}=W_{h} \times V_{h}$ where $W_{h} \subset L^{2}(\Omega)$ is used to approximate the concentration and consists of scalar functions that are constant in each triangle $T_{i}$. For approximating the flux variables, we use the space $V_{h}$. This space is defined over the triangulation $\mathcal{T}_{h}$ as

$$
V_{h}=\left\{v \in V: \forall T \in \mathcal{T}_{h},\left.v_{h}\right|_{T}=\varepsilon+(x, y) \beta, \varepsilon \in \mathbb{R}^{2}, \beta \in \mathbb{R}\right\},
$$

This means that the flux is represented by piecewise linear functions with continuous normal components across the interior boundaries of $\mathcal{T}_{h}$.

The MFE approximation for the solution $v \in V, p \in L^{2}(\Omega)$ of (18), (19) is obtained by solving the finite dimensional problem: find $v_{h} \in V_{h}, p_{h} \in W_{h}$ such that

$$
\left(\nabla \cdot v_{h}, w_{1, h}\right)=\left(q, w_{1, h}\right), \quad \forall w_{1, h} \in W_{h},
$$

and

$$
\left(K^{-1} v_{h}, w_{2, h}\right)-\left(p_{h}, \nabla \cdot w_{2, h}\right)=-\left(p_{D}, w_{2, h} \cdot \eta\right)_{\partial \Omega_{D}}, \quad \forall w_{2, h} \in V_{h} .
$$

Similarly, the MFE approximation for the solution of (20)-(23) is given as follow: find $u_{h}(t) \in W_{h}$, and $z_{h}(t), \tilde{z}_{h}(t) \in V_{h}$ such that

$$
\begin{aligned}
\left(\phi \frac{\partial u_{h}}{\partial t}(t), w_{3, h}\right)+(\nabla & \left.\cdot\left(v u_{h}(t)\right), w_{3, h}\right)+\left(\nabla \cdot z_{h}(t), w_{3, h}\right) \\
& +\int_{0}^{t} K_{e r}(t-s)\left(\nabla \cdot \tilde{z}_{h}(s), w_{3, h}\right) d s=\left(q u_{h}^{*}, w_{3, h}\right)
\end{aligned}
$$

for all $w_{3, h} \in W_{h}$, and

$$
\begin{aligned}
& \left(D_{F}^{-1} z_{h}(t), w_{4, h}\right)-\left(u_{h}(t), \nabla \cdot w_{4, h}\right)=-\left(u_{D}, w_{4, h} . \eta\right)_{\partial \Omega_{D}}, \forall w_{4, h} \in V_{h}, \\
& \left(D_{N F}^{-1} \tilde{z}_{h}(t), w_{5, h}\right)-\left(u_{h}(t), \nabla \cdot w_{5, h}\right)=-\left(u_{D}, w_{5, h} \cdot \eta\right)_{\partial \Omega_{D}}, \forall w_{5, h} \in V_{h}
\end{aligned}
$$

with

$$
u_{h}(0)=u_{0, h}, \quad \text { in } L^{2}(\Omega),
$$

where $u_{0, h}$ is an approximation of $u_{0}$ in $W_{h}$.

In the time integration we use a second-order multistep backward differentiation formula (BDF) method [42] combined with the trapezoidal rule for the integral term. This scheme is applied in a natural implicit-explicit (IMEX) fashion where the non-stiff advection term is treated explicitly and the remain stiff terms are treated implicitly. Let $\Delta t$ be a fixed time step with $t_{n}=n \Delta t$, for $n=0, \ldots, N$, and such that $t_{N}=T$. Denote by $u_{h}^{n} \in W_{h}$ and $z_{h}^{n}, \tilde{z}_{h}^{n} \in V_{h}$ 
the approximations for the solution of (26)-(29) at the time level $t_{n}$. Thus, the proposed IMEX method yields the following approximation

$$
\begin{gathered}
\frac{3}{2 \Delta t}\left(\phi u_{h}^{n+1}, w_{3, h}\right)+\left(\nabla \cdot z_{h}^{n+1}, w_{3, h}\right)+\frac{\Delta t}{2} K_{e r}(0)\left(\nabla \tilde{z}_{h}^{n+1}, w_{3, h}\right)=-\operatorname{sum}(n-1) \\
+\frac{2}{\Delta t}\left(\phi u_{h}^{n}, w_{3, h}\right)-\frac{1}{2 \Delta t}\left(\phi u_{h}^{n-1}, w_{3, h}\right)-\left(\nabla \cdot\left(v u_{h}^{n, n-1}\right)\right. \\
+\left(q^{+} u_{i}, w_{3, h}\right)+\left(q^{-} u_{h}^{n+1}, w_{3, h}\right), \quad \forall w_{3, h} \in W_{h}
\end{gathered}
$$

for $n=1, \ldots, N-1$, and

$$
\begin{aligned}
& \frac{1}{\Delta t}\left(\phi u_{h}^{1}, w_{3, h}\right)+(\nabla\left.\cdot z_{h}^{1}, w_{3, h}\right)+\Delta t K_{e r}(0)\left(\nabla \cdot \tilde{z}_{h}^{1}, w_{3, h}\right)=-\left(\nabla \cdot\left(v u_{h}^{0}\right), w_{3, h}\right) \\
&+\left(q^{+} u_{i}, w_{3, h}\right)+\left(q^{-} u_{h}^{1}, w_{3, h}\right), \quad \forall w_{3, h} \in W_{h},
\end{aligned}
$$

coupled with

$$
\begin{aligned}
& \left(D_{F}^{-1} z_{h}^{n+1}, w_{4, h}\right)-\left(u_{h}^{n+1}, \nabla \cdot w_{4, h}\right)=-\left(u_{D}, w_{4, h} . \eta\right)_{\partial \Omega_{D}}, \quad \forall w_{4, h} \in V_{h}, \\
& \left(D_{N F}^{-1} \tilde{z}_{h}^{n+1}, w_{5, h}\right)-\left(u_{h}^{n+1}, \nabla \cdot w_{5, h}\right)=-\left(u_{D}, w_{5, h} \cdot \eta\right)_{\partial \Omega_{D}}, \quad \forall w_{5, h} \in V_{h},
\end{aligned}
$$

for $n=0, \ldots, N-1$, where

$$
u_{h}^{0}=u_{0, h}, \quad \text { in } L^{2}(\Omega) .
$$

In (30), we have used the relation $q u_{h}^{n+1}=q^{+} u_{i}+q^{-} u_{h}^{n+1}$, with $u_{i}$ the given concentration of injected fluid, $q^{+}=\max \{q, 0\}$ the source term and $q^{-}=$ $\min \{q, 0\}$ the sink term. We also used the notation $u_{h}^{n, n-1}=2 u_{h}^{n}-u_{h}^{n-1}$ and

$$
\operatorname{sum}(n-1)=\frac{\Delta t}{2} \sum_{j=0}^{n-1}\left(K_{e r}\left(t_{n+1}-t_{j+1}\right) \nabla \cdot z_{h}^{j+1}+K_{e r}\left(t_{n+1}-t_{j}\right) \nabla \cdot z_{h}^{j}\right) .
$$

The discretization (31) is obtained by the one-step IMEX Euler method combined with the rectangular rule.

For the particular kernel $K_{e r}(s)=\frac{1}{\tau} e^{-\frac{s}{\tau}}$, the method (30) can be rewritten as the following three-time-level method: for $n=2, \ldots, N-1$,

$$
\begin{aligned}
\frac{3 \tau}{\Delta t^{2}}\left(\phi u_{h}^{n+1}, w_{3, h}\right)-\frac{2 \tau}{\Delta t}\left(q^{-}\right. & \left.u_{h}^{n+1}, w_{3, h}\right)+\frac{2 \tau}{\Delta t}\left(\nabla \cdot z_{h}^{n+1}, w_{3, h}\right) \\
+\left(\nabla \cdot \tilde{z}_{h}^{n+1}, w_{3, h}\right) & =\left(G_{h}^{n}, w_{3, h}\right), \quad \forall w_{3, h} \in W_{h},
\end{aligned}
$$

with

$$
\begin{aligned}
\Delta t^{2} G_{h}^{n}=\tau \phi & \left(4 u_{h}^{n}-u_{h}^{n-1}\right)-2 \tau \Delta t \nabla \cdot\left(v u_{h}^{n, n-1}-e^{-\frac{\Delta t}{\tau}} v u_{h}^{n-1, n-2}\right) \\
+ & \tau \phi e^{-\frac{\Delta t}{\tau}}\left(3 u_{h}^{n}-4 u_{h}^{n-1}+u_{h}^{n-2}\right)+e^{-\frac{\Delta t}{\tau}}\left(\Delta t^{2} \nabla \cdot \tilde{z}_{h}^{n}+2 \tau \Delta t \nabla \cdot z_{h}^{n}\right) \\
& -2 \tau \Delta t e^{-\frac{\Delta t}{\tau}}\left(q^{+} u_{i}+q^{-} u_{h}^{n}\right)+2 \tau \Delta t q^{+} u_{i},
\end{aligned}
$$


and where $u_{h}^{1}$ is given by (31) and $u_{h}^{2}$ is computed from (30) as

$\frac{3 \tau}{\Delta t^{2}}\left(\phi u_{h}^{2}, w_{3, h}\right)-\frac{2 \tau}{\Delta t}\left(q^{-} u_{h}^{2}, w_{3, h}\right)+\frac{2 \tau}{\Delta t}\left(\nabla \cdot z_{h}^{2}, w_{3, h}\right)+\left(\nabla \cdot \tilde{z}_{h}^{n+1}, w_{3, h}\right)=\left(G_{h}^{1}, w_{3, h}\right)$,

for all $w_{3, h} \in W_{h}$, and with

$$
G_{h}^{1}=\frac{2 \tau}{\Delta t}\left(q^{+} u_{i}-\nabla \cdot\left(v u_{h}^{1,0}\right)\right)-2 e^{-\frac{\Delta t}{\tau}} \nabla \cdot \tilde{z}_{h}^{1}-e^{-\frac{2 \Delta t}{\tau}} \nabla \cdot \tilde{z}_{h}^{0}+\frac{\tau \phi}{\Delta t^{2}}\left(4 u_{h}^{1}-u_{h}^{0}\right),
$$

and $\tilde{z}_{h}^{0}=-D_{N F} \nabla u_{h}^{0}$.

It is well known that in this kind of problems advection is predominant over diffusion, therefore, to deal with the advection term in (26), it is important to consider a numerical technique capable of capturing shocks and discontinuities without non-physical oscillations. Here we make use of the second order Monotonic Upstream-Centered Scheme for Conservation Laws (MUSCL), as described in [43]. Schemes that combine MFEs and higher-order Godunov methods, called Godunov-mixed methods, have been successfully applied in the simulation of Fickian transport in porous media [36, 38, 44].

We finish this section with some discussion on the numerical procedure. One major drawback of our method is the stability condition that limits the time step, since we expect this to be determined by the CFL restriction coming from the explicit treatment of advection. It is given by

$$
\Delta t \leq \frac{5}{24} \frac{\phi T_{\min }}{\|v\|_{L^{\infty}}}
$$

where $\|\cdot\|_{L^{\infty}}$ represents the norm of the essential supreme and $T_{\min }$ denotes the minimum ratio between the measure and the perimeter of the triangles in the triangulation $\mathcal{T}_{h}$ [45]. In order to solve the linear systems arising from (26), (27) and (35) we use decomposition techniques based on the Schur complement method. The resulting linear systems are solved by the conjugate gradient method with diagonal preconditioning. This procedure is efficient for (35), since all the matrices envolve are symmetric positive definite and well-conditioned. However, the matrix involved in the flow system (26), (27) is strongly indefinite [40] and it is well-known that iterative methods for indefinite systems are not so efficient as those for problems with positive definite matrices. Many different approaches have been proposed to address this issue, and without being exhaustive, we refer to $[46,47,48,49,50]$. Finally, we note that in this work we focus on triangular elements, as they provide the greater geometric flexibility, a crucial aspect in porous media simulation, allied with good stability and convergence properties. Moreover, the proposed method can easily incorporate mesh adaptive procedures.

\subsection{Numerical study of convergence}

In this section we present some numerical results to test the accuracy of the proposed method using problems with known analytical solutions. All problems 
are set on the unit square $\Omega=(0,1)^{2}$. The numerical error and the convergence rates were obtained on a sequence of five mesh refinements. The initial mesh, shown in Figure 3, was generated by a conformed Delaunay triangulation.

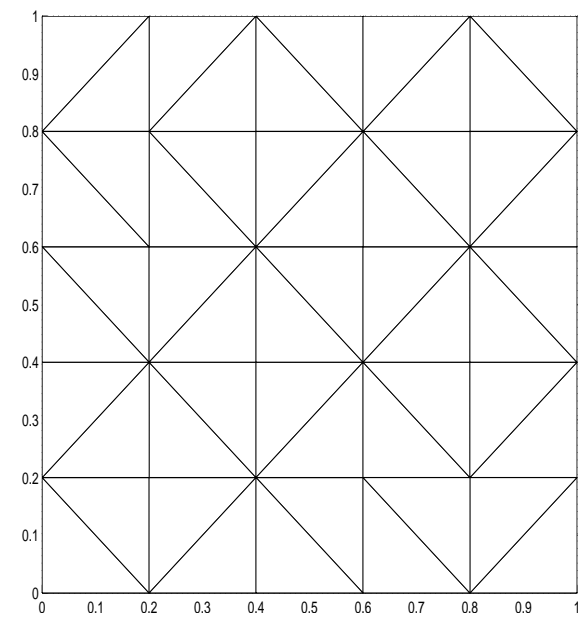

Figure 3: Initial Delaunay mesh.

The concentration and pressure errors are measured by the $L^{2}(\Omega)$ discrete norm

$$
\left\|w_{h}\right\|_{h}^{2}=\sum_{n=1}^{N_{T}}\left|T_{n}\right|\left(w_{h}\left(x_{T_{n}}\right)\right)^{2},
$$

where $x_{T_{n}}$ denotes the centroid of the triangle $T_{n}$, while the flux error is calculated with the $H($ div $; \Omega)$ discrete norm

$$
\left\|w_{h}\right\|_{d i v, h}^{2}=\left\|w_{h}\right\|_{0, h}^{2}+\left\|w_{h}\right\|_{1, h}^{2},
$$

with

$$
\begin{aligned}
\left\|w_{h}\right\|_{0, h}^{2} & =\sum_{n=1}^{N_{T}}\left|T_{n}\right| \sum_{i=1}^{3}\left(w_{h}\left(x_{E_{i}}\right) \cdot \eta_{E_{i}}\right)^{2}, \\
\left\|w_{h}\right\|_{1, h}^{2} & =\sum_{n=1}^{N_{T}} \sum_{i=1}^{3}\left|E_{i}\right|\left(w_{h}\left(x_{E_{i}}\right) \cdot \eta_{E_{i}}\right)^{2},
\end{aligned}
$$

where $E_{i}$, for $i=1,2,3$, represents the edges of $T_{n}$ and $x_{E_{i}}$ stands for the midpoint of the edge $E_{i}$.

With the first two examples, we intent to analyze the numerical error of the spatial discretization of (9).

Example 1 In this example we consider $q=0, \phi=1, \tau=1, T=0.5$ and the fixed time step $\Delta t=5 \times 10^{-4}$. The parameters for the integro-differential 
equation (9) are

$$
v=0, \quad D_{m}=\left[\begin{array}{cc}
0.25 & 0.1 \\
0.1 & 0.2
\end{array}\right], \quad \text { and } \quad D_{d}=\left[\begin{array}{cc}
0.5 & 0.1 \\
0.1 & 0.4
\end{array}\right] .
$$

The remaining undefined terms are such that it has the solution

$$
u(x, y, t)=e^{2 t} x y(x-1)(y-1) \sin (x y) .
$$

In Example 1, where advection is not present, the convergence rate should be governed by the mixed method. Therefore, we predict a convergence rate equal to two for the scalar variable in the norm (38) and a convergence rate equal to one for the flux variable in the norm $\|\cdot\|_{d i v, h}$. This expectations are

Table 3: Discrete norm errors and numerical convergence rates for Example 1.

\begin{tabular}{llllll}
\hline$\left\|u-u_{h}\right\|_{h}$ & Rate & $\left\|\tilde{z}-\tilde{z}_{h}\right\|_{d i v, h}$ & Rate & $\left\|z-z_{h}\right\|_{d i v, h}$ & Rate \\
\hline $1.2358 \times 10^{-3}$ & 1.6878 & $3.1111 \times 10^{-2}$ & 1.1254 & $1.9625 \times 10^{-2}$ & 9.2069 \\
$3.8358 \times 10^{-4}$ & 1.8436 & $1.4260 \times 10^{-2}$ & 1.1326 & $1.0367 \times 10^{-2}$ & 1.1035 \\
$1.0688 \times 10^{-4}$ & 1.9323 & $6.5040 \times 10^{-3}$ & 1.1193 & $4.8247 \times 10^{-3}$ & 1.2490 \\
$2.8002 \times 10^{-5}$ & 1.9736 & $2.9939 \times 10^{-3}$ & 1.0778 & $2.0300 \times 10^{-3}$ & 1.2949 \\
$7.1296 \times 10^{-6}$ & - & $1.4183 \times 10^{-3}$ & - & $8.2735 \times 10^{-4}$ & - \\
\hline
\end{tabular}

based on known results for second-order elliptic problems [51, 52, 53, 41]. We observe in Table 3 that the numerical convergence rates are in agreement with our predictions.

Example 2 In this example, we consider equation (9), with

$$
v=(1,1) \quad \text { and } \quad D_{f}=D_{N F}=q=0 .
$$

The initial condition is defined as

$$
c_{0}(x, y)=\sin (2 \pi x) \sin (2 \pi y)
$$

and periodic boundary conditions are imposed. The solution for this problem is then given by

$$
c(x, y, t)=\sin (2 \pi(x-T)) \sin (2 \pi(y-T)) .
$$

The time step is the maximum allowed by the CFL condition (37), and the results for $T=0.1$ are shown in Table 4. For comparison, we also present the results obtained with the upwind method, which we identify with the subscript up.

In Example 2, dispersion is null; therefore, the error is dominated by the MUSCL method. Depending on the problem, we could expect this numerical scheme to have an order of accuracy between one and two. However, it is a 
Table 4: Discrete norm errors and numerical convergence rates for Example 2.

\begin{tabular}{llll}
\hline$\left\|c-c_{h}\right\|_{h}$ & Rate & $\left\|c-c_{h, u p}\right\|_{h}$ & Rate \\
\hline $1.2997 \times 10^{-1}$ & 1.0328 & $1.9471 \times 10^{-1}$ & 0.65960 \\
$6.3523 \times 10^{-2}$ & 1.4492 & $1.2326 \times 10^{-1}$ & 0.77846 \\
$2.3264 \times 10^{-2}$ & 1.4861 & $7.1861 \times 10^{-2}$ & 0.86229 \\
$8.3047 \times 10^{-3}$ & 1.5206 & $3.9529 \times 10^{-2}$ & 0.90935 \\
$2.8946 \times 10^{-3}$ & - & $2.1046 \times 10^{-2}$ & - \\
\hline
\end{tabular}

well-known fact that second-order MUSCL schemes like the one proposed here rarely, if ever, achieve second-order convergence rate. Nevertheless, the option for higher-order MUSCL methods over first-order upwind scheme is justified since they present less numerical dispersion, smaller error and higher-order of accuracy $[54,55,56]$. The results exhibit in Table 4 confirm these predictions.

Example 3 In this example we analyze the method (24), (25) for flow system (18), (19) with a full tensor, defined by

$$
K=\left[\begin{array}{cc}
(x+1)^{2}+y & \sin (x y) \\
\sin (x y) & 2
\end{array}\right]
$$

and equation (18) with a nonhomogeneous second member such that this flow problem admits the solution

$$
p(x, y)=x+y+\sin (x y) \cos (y) .
$$

The boundary conditions are of Neumann type on $x=1$ and $y=1$ and of Dirichlet type on $x=0$ and $y=0$.

In Example 3, we test the numerical method for solving the flow system (18), (19). The numerical results are given in Table 5, and they are as expected. The pressure error $\left\|p-p_{h}\right\|_{h}$ presents second-order convergence while $\left\|v-v_{h}\right\|_{d i v, h}$ and $\left\|v-v_{h}\right\|_{0, h}$ present first- and second-order convergence, respectively.

Table 5: Discrete norm errors and numerical convergence rates for Example 3.

\begin{tabular}{llllll}
\hline$\left\|p-p_{h}\right\|_{h}$ & Rate & $\left\|v-v_{h}\right\|_{d i v, h}$ & Rate & $\left\|v-v_{h}\right\|_{0, h}$ & Rate \\
\hline $4.0019 \times 10^{-3}$ & 1.9822 & $1.6759 \times 10^{-1}$ & 1.2358 & $4.7619 \times 10^{-2}$ & 1.7009 \\
$1.0129 \times 10^{-3}$ & 1.9915 & $7.1160 \times 10^{-2}$ & 1.2850 & $1.4647 \times 10^{-2}$ & 1.7703 \\
$2.5473 \times 10^{-4}$ & 1.9966 & $2.9203 \times 10^{-2}$ & 1.3443 & $4.2939 \times 10^{-3}$ & 1.8367 \\
$6.3832 \times 10^{-5}$ & 1.9989 & $1.1502 \times 10^{-2}$ & 1.3752 & $1.2021 \times 10^{-3}$ & 1.8713 \\
$1.5971 \times 10^{-5}$ & - & $4.4340 \times 10^{-3}$ & - & $3.2858 \times 10^{-4}$ & - \\
\hline
\end{tabular}

Example 4 In this last example, we examine the rate of convergence in time. In order to do that, we consider the problem of Example 1, but with $v=(1,0.5)$ 
and $T=3$. We successively solve this problem, for different time steps, in the refinement level corresponding to the last row of Tables 3, 4 and 5. The results obtained are presented in Table 6 and they indicate that the time integration scheme is second-order accurate.

Table 6: Discrete norm error and estimated order of accuracy in time.

\begin{tabular}{lll}
\hline$\Delta t$ & $\left\|u-u_{h}\right\|_{h}$ & Rate \\
\hline $5.0000 \times 10^{-1}$ & $2.7460 \times 10^{-1}$ & 1.2872 \\
$2.5000 \times 10^{-1}$ & $1.1251 \times 10^{-1}$ & 1.6416 \\
$1.2500 \times 10^{-1}$ & $3.6062 \times 10^{-2}$ & 1.8555 \\
$6.2500 \times 10^{-2}$ & $9.9650 \times 10^{-3}$ & 2.0259 \\
$3.1250 \times 10^{-2}$ & $2.4470 \times 10^{-3}$ & - \\
\hline
\end{tabular}

\subsection{Numerical experiments}

After the validation of the proposed numerical method, we present in this section some more realistic examples of tracer transport in porous media. A typical porous medium is characterized by rapid changes in the permeability. Therefore, in the following examples, we attempted to replicate this situation. We consider $\Omega=(0,1)^{2}$ where a conforming Delaunay triangulation with 3200 elements is introduced and the time step is taken in accordance with the CFL restriction (37).
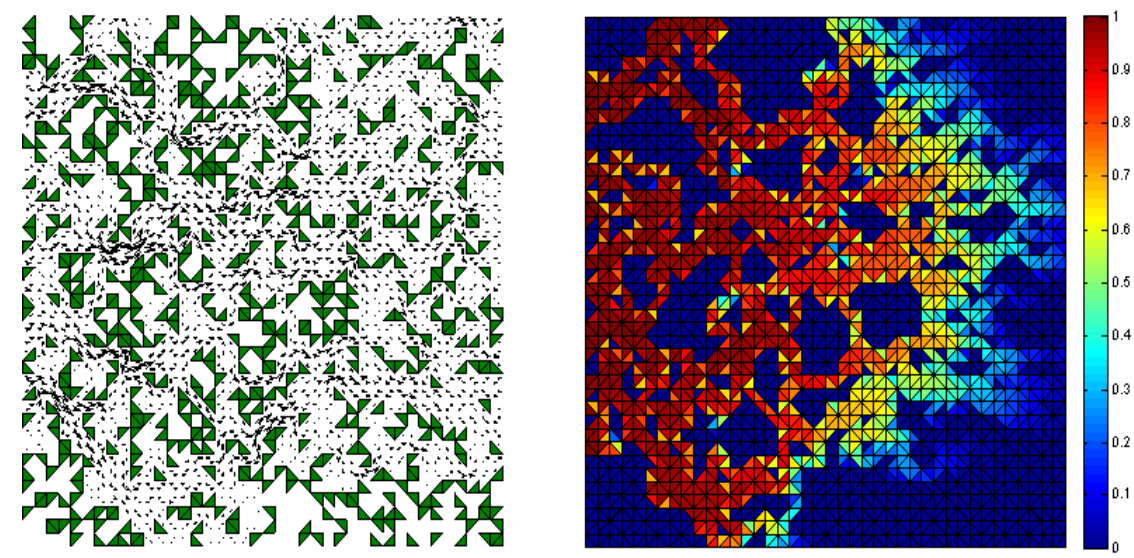

Figure 4: Computed velocity (left) and concentration profile (right).

For the first example, we consider the permeability field shown in Figure 4. It consists of two zones that differ in six orders of magnitude: the white zone has a high permeability, $K=I$, while the green zone has a very low permeability, 
$K=10^{-6} I$. No flow conditions are imposed at the horizontal boundaries, while in the vertical boundaries the pressure is set $p=1$ and $p=0.1$ at the left and right boundaries, respectively. The resulting velocity field is also shown in Figure 4 and it seems to represent very well the heterogeneities pattern. We also consider a tracer transport process that obeys the equation (9). We define the tensors $D_{F}=D_{N F}$ as in (2), with the molecular diffusion $d_{m}=0$ and the dispersivities $\alpha_{\ell}=5 \times 10^{-4}$ and $\alpha_{t}=2 \times 10^{-5}$. We take the porosity $\phi=0.3$, the parameter $\tau=10$, and no source or sink terms $q=0$. We impose zero initial condition for concentration, $u=1$ on the left boundary, $u=0$ on the right boundary, and impermeable conditions on the remaining ones. In Figure 4, we observe that the concentration profile is in good agreement with that expected from the proposed permeability field. Note that the low permeability zones are not invaded by the tracer.

Now, we consider a transport problem with a source and a sink term. The source covers one triangle near the lower-left corner with a rate of $q=6.4$. The injected tracer concentration $u_{i}$ is equal to one. The sink also covers one triangle and is located near the upper-right corner with an opposite rate to the source. No flow boundary conditions are assumed both for velocity and concentration. Also, we take $\tau=50, \phi=0.3$, and $D_{F}=D_{N F} / 10$ defined as in (2) with $d_{m}=10^{-6}, \alpha_{\ell}=4 \times 10^{-3}$, and $\alpha_{t}=2 \times 10^{-3}$. The random permeability field is shown in Figure 5 (a) and was generated using a Gaussian distribution. As we can see, there are jumps of about four orders of magnitude throughout the domain. The computed velocity field and the estimated tracer concentration at $T=30$ and $T=60$ are also presented in Figure 5. Again, the numerical results seem consistent with respect to the permeability field.
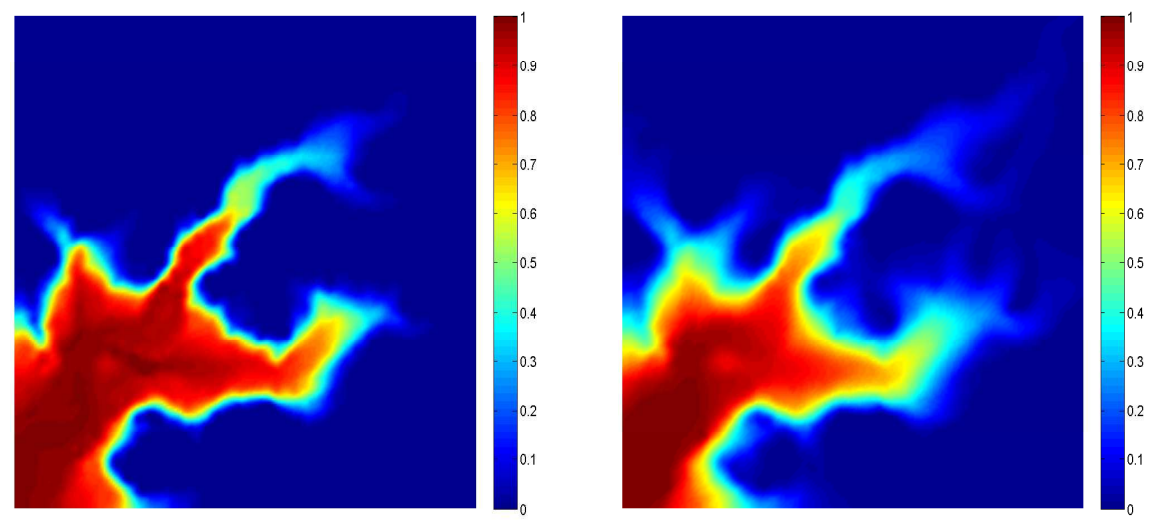

Figure 6: Concentration at $T=30$ for $\tau=50$ (left) and $\tau=0.1$ (right).

Finally, with the aim of testing the effect of the parameter $\tau$, we solved the same problem but with $\tau=0.1$. In Figure 6 , we display the concentration profiles at $T=30$ for the two different scenarios. For the lower value of $\tau$, the 


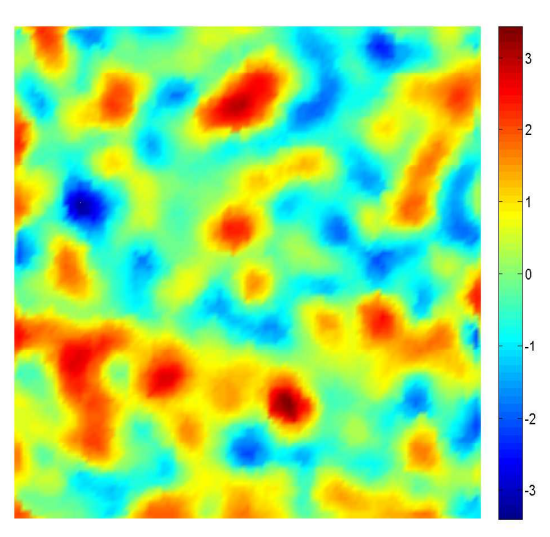

(a)

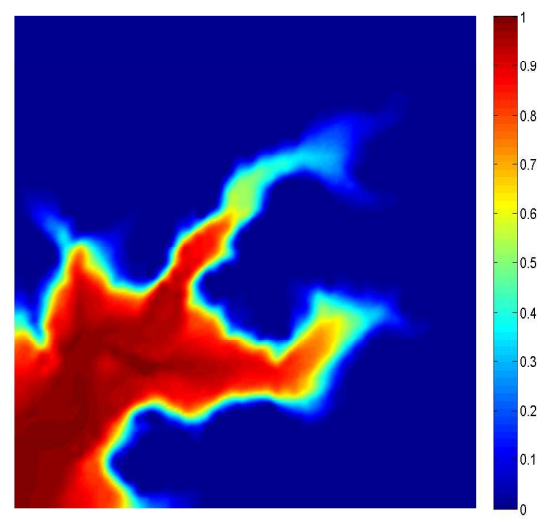

(c)

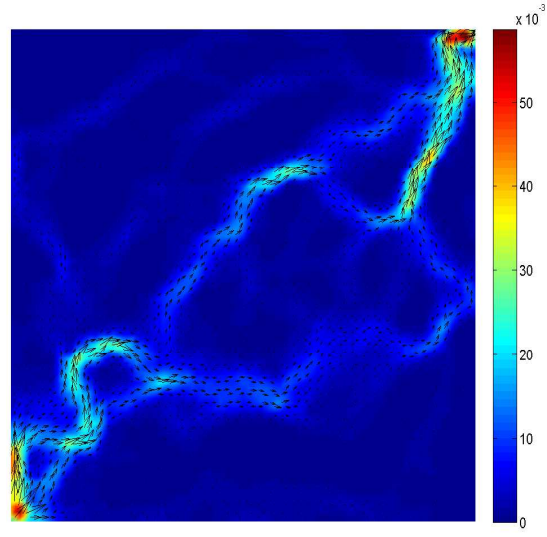

(b)

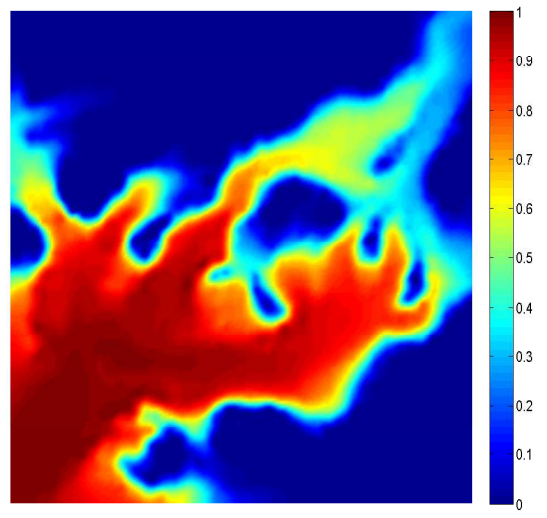

(d)

Figure 5: The log-permeability field (a), vectors and Euclidean norm of the velocity field (b), the concentration at $T=30$ (c) and the concentration at $T=60(\mathrm{~d})$.

transport seems to be more dispersive, the plume of tracer is less compressed and more widespread.

\section{Conclusions}

In this work, we proposed and tested an IDM to describe non-Fickian tracer movement. The results presented for the one-dimensional version suggest that this model can overcome the limitations of the traditional Fickian ADE. These findings confirm the results already obtained by the authors in [27]. A numerical scheme for the two-dimensional model, using a Godunov-mixed method in space 
and a second-order IMEX integrator in time, was also developed. To validate the code, we performed some numerical experiments, including comparisons with analytical solutions. We saw that the method was accurate and generated numerical solutions that were stable and physically reasonable. These results prove the computational feasibility of the proposed non-Fickian model.

\section{Acknowledgments}

This work was partially supported by the Centro de Matemática da Universidade de Coimbra (CMUC), funded by the European Regional Development Fund through the program COMPETE and by the Portuguese Government through the FCT - Fundação para a Ciência e Tecnologia under the projects PEstC/MAT/UI0324/2013 and UTAustin/MAT/0066/2008.

\section{References}

[1] Douglas J. Superconvergence in the pressure in the simulation of miscible displacement. SIAM J. Numer. Anal. 1985; 22:962-969.

[2] Ewing R, Wheeler M. Galerkin method for miscible displacement problems in porous media. SIAM J. Numer. Anal. 1980; 17:351-365.

[3] Peaceman D. Improved treatment of dispersion in numerical calculation of multidimensional miscible displacement. Soc. Pet. Eng. J. 1996; 6:213-216.

[4] Ewing R. The mathematics of reservoir simulation. SIAM, 1984.

[5] Settari A, Price H, Dupont T. Development and application of variational methods for simulation of miscible displacement in porous media. Soc. Pet. Eng. J. 1977; 17:228-246.

[6] Chen HT, Liu KC. Analysis of non-Fickian diffusion problems in a composite medium. Comput. Phys. Comm. 2003; 150:31-42.

[7] Hassanizadeh S. On the transient non-Fickian dispersion theory. Transport Porous Med. 1996; 23:107-124.

[8] Neuman S, Tartakovsky D. Perspective on theories of non-Fickian transport in heterogeneous media. Adv. Water Resour. 2009; 32:670-680.

[9] Bromly M, Hinz C. Non-Fickian transport in homogeneous unsaturated repacked sand. Water Resour. Res. 2004; 40.

[10] Cortis A, Berkowitz B. Anomalous transport in "classical" soil and sand columns. Soil Sci. Soc. Am. J. 2004; 68:1539-1548.

[11] Cortis A, Chen Y, Scher H, Berkowitz B. Quantitative characterization of pore-scale disorder effects on transport in "homogeneous" granular media. Phys. Rev. E 2004; 70:041 108. 
[12] Gelhar L, Welty C, Rehfeldt K. A critical review of data on field-scale dispersion in aquifers. Water Resour. Res. 1992; 28:1955-1974.

[13] Huang K, Toride N, van Genuchten M. Experimental investigation of solute transport in large, homogeneous and heterogeneous, saturated soil columns. Transport Porous Med. 1995; 18:283-302.

[14] Levy M, Berkowitz B. Measurement and analysis of non-Fickian dispersion in heterogeneous porous media. J. Contam. Hydrol. 2003; 64:203-206.

[15] Mackay D, Freyberg D. A natural gradient experiment on solute transport in a sand aquifer: 1. Approach and overview of plume movement. Water Resour. Res. 1986; 22:2017-2029.

[16] Sidle R, Nilsson B, Hansen M, Fredericia J. Spatially varying hydraulic and solute transport characteristics of a fractured till determined by field tracer tests, Funen, Denmark. Water Resour. Res. 1998; 34:2515-2527.

[17] Silliman S, Konikow L, Voss C. Laboratory investigation of longitudinal dispersion in anisotropic porous media. Water Resour. Res. 1987; 23:21452151.

[18] Sternberg S, Cushman J, Greenkorn R. Laboratory observation of nonlocal dispersion. Transport Porous Med. 1996; 23:135-151.

[19] Strack O. A mathematical model for dispersion with a moving front in groundwater. Water Resour. Res. 1992; 28:2973-2980.

[20] Barbeiro S, Ferreira J, Pinto L. $H^{1}$ - second order convergent estimates for non-Fickian models. Appl. Numer. Math. 2011; 61:201-215.

[21] Cannon J, Lin Y. A priori $L^{2}$ error estimates for finite-element methods for nonlinear diffusion equations with memory. SIAM J. Numer. Anal. 1990; 27:595-607.

[22] Ewing R, Lazarov R, Lin Y. Finite volume element approximations of nonlocal in time one-dimensional flows in porous media. Computing 2000; 64:157-182.

[23] Ewing R, Lin Y, Sun T, Wang J, Zhang S. Sharp $L^{2}$-error estimates and superconvergence of mixed finite element methods for non-Fickian flows in porous media. SIAM J. Numer. Anal. 2002; 40:1538-1560.

[24] Ferreira J, Gudiño E, de Oliveira P. A second order approximation for quasilinear non-Fickian models. Comput. Meth. Appl. Math. 2013; 13:471493.

[25] Ferreira J, Pinto L, Romanazzi G. Supraconvergence and supercloseness in Volterra equations. Appl. Numer. Math. 2012; 62:1718-1739. 
[26] Sinha R, Ewing R, Lazarov R. Some new error estimates of a semidiscrete finite volume element method for a parabolic integro-differential equation with nonsmooth initial data. SIAM J. Numer. Anal. 2006; 43:2320-2344.

[27] Ferreira J, Pinto L. Non-Fickian tracer transport in porous media. Proceedings of the 14th International Conference on Computational and Mathematical Methods in Science and Engineering, 2014.

[28] Dentz M, Cortis A, Scher H, Berkowitz B. Time behavior of solute transport in heterogeneous media: transition from anomalous to normal transport. Adv. Water Resour. 2004; 27:155-173.

[29] Gao G, Zhan H, Feng S, Huang G, Mao X. Comparison of alternative models for simulating anomalous solute transport in a large heterogeneous soil column. J. Hydrol. 2009; 377:391-404.

[30] Cortis A, Berkowitz B. Computing "anomalous" contaminant transport in porous media: the CTRW MATLAB toolbox. Ground Water 2005; 43:947950.

[31] Scheidegger A. An evaluation of the accuracy of the diffusivity equation for describing miscible displacement in porous media. Proceedings of Theory of Fluid Flow in Porous Media, 1959.

[32] Huang G, Huang Q, Zhan H. Evidence of one-dimensional scale-dependent fractional advection-dispersion. J. Contam. Hydrol. 2006; 85:53-71.

[33] Logan J. Solute transport in porous media with scale-dependent dispersion and periodic boundary conditions. J. Hydrol. 1996; 184:261-276.

[34] Mishra S, Parker J. Analysis of solute transport with a hyperbolic scaledependent dispersion model. Hydrol. Process. 1990; 4:45-57.

[35] Zoua S, Mab J, Koussisc A. Analytical solutions to non-Fickian subsurface dispersion in uniform groundwater flow. J. Hydrol. 1996; 179:237-258.

[36] Arbogast T, Bryant S, Dawson C, Saaf F, Wang C, Wheeler M. Computational methods for multiphase flow and reactive transport problems arising in subsurface contaminant remediation. J. Comput. Appl. Math. 1996; 74:19-32.

[37] Arbogast T, Huang C. A fully mass and volume conserving implementation of a characteristic method for transport problems. SIAM J. Sci. Comput. 2066; 28:2001-2022.

[38] Bergamaschi L, Mantica S, Manzini G. A mixed finite element-finite volume formulation of the black-oil model. SIAM J. Sci. Comput. 1998; 20:970997.

[39] Lipnikov K, Shashkov M, Yotov I. Local flux mimetic finite difference methods. Numer. Math. 2009; 112:115-152. 
[40] Brezzi F, Fortin M. Mixed and hybrid finite element methods. SpringerVerlag New York, 1991.

[41] Raviart P, Thomas J. A mixed finite element method for 2-nd order elliptic problems. Mathematical Aspects of Finite Element Methods, Lecture Notes in Mathematics 1977; 606:292-315.

[42] Crouzeix M. Une méthode multipas implicite-explicite pour l'approximation des équations d'évolution paraboliques. Numer. Math. 1980; 35:257-276.

[43] Barth T, Jespersen D. The design and application of upwind schemes on unstructured meshes. AIAA paper 93-0668 1993; .

[44] Dawson C. Godunov mixed methods for immiscible displacement. Internat. J. Numer. Methods Fluids 1990; 11:835-847.

[45] Hundsdorfer W, Ruuth S, Spiteri R. Monotonicity-preserving linear multistep methods. SIAM J. Numer. Anal. 2003; 41:605-623.

[46] Ewing R, Lazarov R, Pasciak J, Vassilev A. Mathematical modeling, numerical techniques, and computer simulation of flows and transport in porous media. Proceedings of Computational Techniques and Applications, 1995.

[47] Bramble J, Pasciak J, Vassilev A. Analysis of the inexact Uzawa algorithm for saddle point problems. SIAM J. Numer. Anal. 1997; 34:1072-1092.

[48] Brenner S. A multigrid algorithm for the lowest-order Raviart-Thomas mixed triangular finite element method. SIAM J. Numer. Anal. 1992; 29:647-678.

[49] Arbogast T, Dawson C, Keenan P, Wheeler M, Yotov I. Enhanced cellcentered finite differences for elliptic equations on general geometry. SIAM J. Sci. Comput. 1998; 19:404-425.

[50] Arbogast T, Dawson C, Keenan P. Efficient mixed methods for groundwater flow on triangular or tetrahedral meshes. Water Trans. 1994; 1:3-10.

[51] Acosta G, Duran RG. The maximum angle condition for mixed and nonconforming elements: Application to the Stokes equations. SIAM J. Numer. Anal. 2000; 37:18-36.

[52] Douglas J, Roberts J. Global estimates for mixed methods for second order elliptic equations. Math. Comp. 1985; 44:39-52.

[53] Gastaldi L, Nochetto R. Optimal $L^{\infty}$-error estimates for nonconforming and mixed finite element methods of lowest order. Numer. Math. 1987; 50:587-611.

[54] Hubbard M. Multidimensional slope limiters for MUSCL-type finite volume schemes on unstructured grids. J. Comput. Phys. 1999; 155:54-74. 
[55] Buffard T, Clain S. Monoslope and multislope MUSCL methods for unstructured meshes. J. Comput. Phys. 2010; 229:3745-3776.

[56] Park J, Yoon SH, Kim C. Multi-dimensional limiting process for hyperbolic conservation laws on unstructured grids. J. Comput. Phys. 2010; 229:788812. 